\title{
Simulation and Performance Testing of the Network Control System in Coal Mine Automation
}

\author{
Wu Haiyan ${ }^{1,2, a}$, Zhang Xuhui ${ }^{1,2, a}$, Zhao Youjun ${ }^{2, b}$ and Lou Linli ${ }^{2, b}$ \\ ${ }^{1}$ School of Mechanical Engineering, Xi'an University of Science and Technology, Xi'an 710054, \\ China \\ ${ }^{2}$ Xi'an Coal Mining Machinery Co., Ltd., Xi'an 710032, China \\ axust_zxh@163.com, bjixie3000@163.com
}

\begin{abstract}
Keywords: Network Control System; Coal Mining equipment; Real-time performance; Simulation research; TrueTime

Abstract. Aiming at the problems of real-time and reliability of the Network Control System(NCS) in coal mine automation, the performance of monitoring system based on Industrial Ethernet is studied. Through theoretical analysis and computer simulation, the real time performance including network delay, sampling rate, information transfer interval of the system were analyzed with the help of Jitterbug and TrueTime toolbox. Optimize the parameter of system communication network performance by comprehensive evaluation method, system optimal operation parameters was got, improved the reliability and safety of equipment.
\end{abstract}

\section{Introduction}

Network system during modern coal mine production is not only the bound of the coal mine enterprises' information, but also the indispensable part of unmanned mining in fully mechanized coal mining face[1]. The control system in distributed environment is composed of the controlled object, sensor, controller, actuator, and digital communication network. The detection, control, coordination and instruction of the system are transmitted through the communication network. And functions such as yet estimating, control and diagnosis function may be executed distributive in the different network nodes. According to the different communication network conditions, time delay, packet loss and other phenomena have a great influence on the performance of the system which is controlled by the internet. If these problems are not concerned in the system design analysis, the control ability will be lowered down and finally causes the instability of the system [2-3]. So when the Internet is used in real-time control, researchers are more concerned about the system ability under the network environment.

By using the methods of minimizing the conflict domain, controlling the flow, improving the performance of the intelligent device or communication processing unit, the real time communication protocol and other measures can be guaranteed [4]. But because of the complexion in both time and space, there are difficulties in the network distribution and node data acquisition. Establish a test-bed is limited, easy to be damage, difficult to reuse, and is too expensive, besides it is hard to evaluated the performance of the network control system quantitatively. Using the software stimulation online to analysis, search and design the control system is an efficient and economic subsidiary mean[5].This paper uses the simulation method to study the real time performance including network delay, sampling rate, information transfer interval of the system to increase the reliability and timeliness of the coal mine equipment.

\section{Modeling Analysis of the Network Control System}

The typical structure of the network control system with time delay is shown in Fig.1,in which the $\mathrm{ZOH}$ represents the zero-order holder, $\tau_{s c}$ is the time delay of the controller, $\tau_{c a}$ is the time delay from the controller to the actuator. To simplify, the calculation of time delay is regarded as $\tau_{c a}$. 


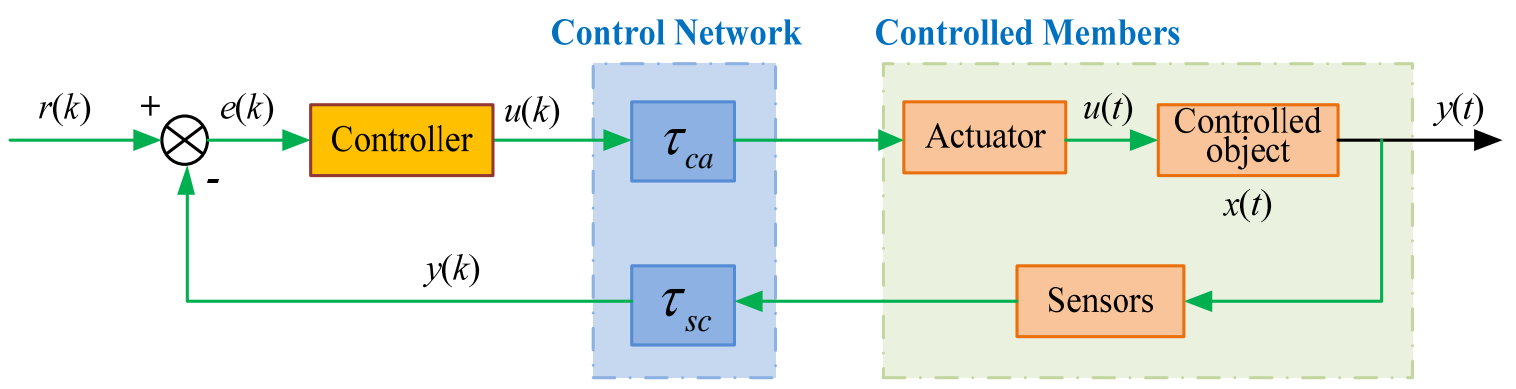

Fig.1 Block diagram of network control system with time delay

Some special protocols can ensure the network delay to be a fixed value like the CAN bus protocol [5], but others are time varying such as the DeviceNet, wireless internet and the Ethernet [6] .One of the method to do constants for the time delay is to create buffer in the receive side, which can simply the process the drawback is that the analysis and design results are conservative due to the transition data are all concerned under the worst circumstance. In many situations in real network control systems, the controller and the driver are placed in the controlled device side so the impact of the data base can be ignored.

Assume that the state equation of the continuous controlled object is:

$$
\boldsymbol{A}(t)=\boldsymbol{A x}(t)+\mathbf{B} \boldsymbol{u}(t)
$$

In which $t \in[k T+\tau,(K+1) T+\tau], \tau<T$

$$
\boldsymbol{y}(t)=\boldsymbol{C} \boldsymbol{x}(t)
$$

The discrete linear control rate is

$$
\boldsymbol{u}\left(t^{+}\right)=-\boldsymbol{K} \boldsymbol{x}(t-\tau) \quad t \in\{k T+\tau, k=0,1,2 \ldots\}
$$

$\boldsymbol{x}(t)$ represents the system state; $\boldsymbol{u}(t)$ represents the control input; $\boldsymbol{y}(t)$ represents the system output;

$\boldsymbol{A} 、 \boldsymbol{B} 、 \boldsymbol{C}$ are matrix with different dimensions.

\section{The impact of the time delay in control systems}

As for the different situations caused by $\tau_{k}$, Xuedong and Pengchen gave some conditions that ensure the stability of the system that can make the value of the solution at the sampling point [7].The buffer is introduced in the actuator and the controller terminal communication module. The random time delay caused by CSMA/CD is turned into a constant time delay. So the stability problem of the network control system can be transformed into the matrix test Schur stability one [8].

The control system with the constant time delay can be concluded to the matrix test (4)for the Schur stability.

$$
H=\left[\begin{array}{cc}
e^{A T} & -E(T) B K \\
e^{A(T-\tau)} & -e^{-A \tau}(E(T)-E(\tau)) B K
\end{array}\right]
$$

In which $E(T)=\int_{0}^{T} e^{A(T-\tau)} B d t$.

In addition to it, the time delay ${ }^{\tau_{s c}}$ can be rectified by the improvement and reasonable detector design and gains the approximation of the object in the timeless delay calculated by the computer.

The estimate value of the controlled object state $x\left(k T+\tau_{s c, k}\right)$ is determined by $k T$ time sample output and the time delay $\tau_{s c}$ between the transducer and controller. When the system is in the full state feedback, observer only need to make compensate for the time delay $\tau_{s c}$, in the control signal producing process, we use this kind of compensation to obtain the more accurate value of the object. Assume that the state equation of the system object is like equation(1) and equation(2), concerning about the controller model, its analytic formula can be summarized as:

$$
\boldsymbol{x}(t)=e^{A t} \boldsymbol{x}(0)+\int_{0}^{t} e^{A(t-s)} \boldsymbol{B} \boldsymbol{u}(s) d s
$$

The time sequence of controlled object and observer in full state feedback shows in figure 2 . Where $\tau_{s c, k}$ represents that when the control state is $\boldsymbol{x}(k T)$ the time delay between the transducer and 
the controller; $\overline{\boldsymbol{x}}\left(k T+\tau_{s c, k}\right)$ is the time state $\boldsymbol{x}(k T)$ experiment observation results. Assume that there is no measurement noise during the sampling process, then according to the formula (4), the state $\boldsymbol{x}(k T)$ has the time delay observe result $\overline{\boldsymbol{x}}\left(k T+\tau_{s c, k}\right)$ which can be calculated by the following formula:

$$
\overline{\boldsymbol{x}}\left(k T+\tau_{s c, k}\right)=\boldsymbol{x}\left(k T+\tau_{s c, k}\right)=e^{A \tau_{s c, k}} \boldsymbol{x}(k T)+\int_{k T}^{k T+\tau_{s c, k}} e^{A\left(k T+\tau_{s, k}-s\right)} \boldsymbol{B} \boldsymbol{u}(s) \mathrm{d} s
$$

The feedback controller of the system is

$$
\boldsymbol{u}\left(k T+\tau_{s c, k}\right)=-\boldsymbol{K} \bar{x}\left(k T+\tau_{s c, k}\right)
$$

According to this control strategy, this closed-loop system can be concluded as:

$$
\left.\boldsymbol{x}\left[(k+1) T+\tau_{k+1}\right]\right) \nRightarrow\left(\phi\left(\delta_{k}\right) \boldsymbol{x}\left(k T+\tau_{k}\right)\right.
$$

In which $\delta_{k}=T+\tau_{s c, k+1}-\tau_{s c, k} ; \Phi\left(\delta_{k}\right)=\Gamma\left(\delta_{k}\right) \boldsymbol{K} ; \boldsymbol{\Phi}\left(\delta_{k}\right)=e^{A \delta_{k}} ; \Gamma\left(\delta_{k}\right)=\int_{0}^{\delta_{k}} e^{A s} \boldsymbol{B} \mathrm{d} s$.

According to the analysis above, in the network control system applied with the time-driven sensors, the time delay $\tau_{s c}$ from the sensor to the controller does estimations by forming the rational detector and then attaining the object state approximation that can be used in system analysis.

Using the Jitterbug tool box to make platform for analysis of time delay network control system,

Make simulation analysis of the static performance for all the time delay conditions in linear closed loop control system. Jitterbug is composed of two important parallel modules, the signal module and the timing module, as is shown in Figure 3.

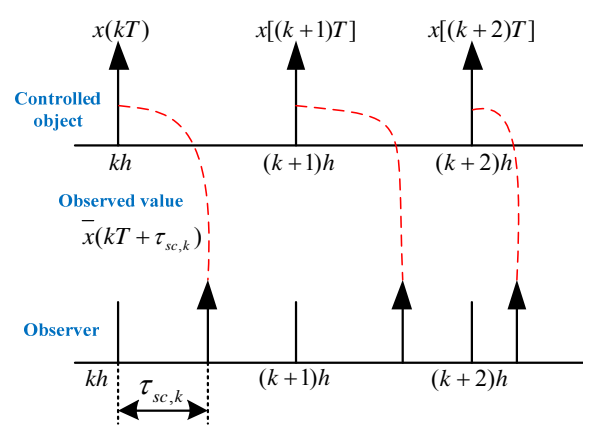

Fig.2. Control sequence in feedback

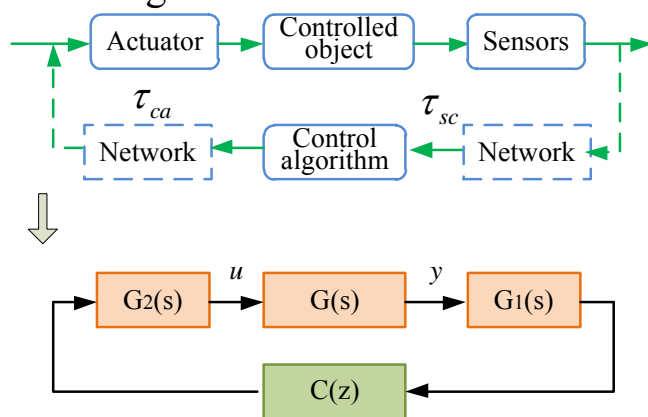

(a) Signal module

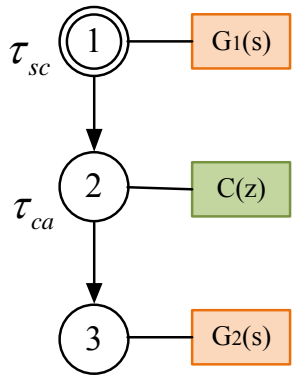

(b)Timing module

Fig.3 Jitterbug Model of NCS

Jitterbug adapts a two order performance evaluation criterion, studies the performance of the system under various time series, which is

$$
J_{C, D}=\lim _{t \rightarrow \infty} \frac{1}{t} \int_{0}^{t}\left|\begin{array}{l}
x_{i}(t) \\
u_{i}(t)
\end{array}\right| Q_{i}\left|\begin{array}{l}
x_{i}(t) \\
x_{i}(t)
\end{array}\right| \mathrm{d} t
$$

The cost function of the total control system through the sum of all the input and output calculations of continuous and discrete systems,

$$
J=\sum J_{C}+\sum J_{D}
$$

Use the DC servo system as the controlled object, the transfer function is $G(s)=1000 / s(s+1)$, the sensors and actuator are of discrete-time system, $G_{1}(z)=G_{2}(z)=1$. The controlled objects is connected with a PID controller through the Internet. In the fixed sampling time period, the sensors take sample from the input signals, then transfer it to the controller to calculate, finally transfer it into the actuator to dispose. As the formula shows:

$$
J=\lim _{t \rightarrow \infty} \frac{1}{T} \int_{0}^{T} y^{2}(t)+u^{2}(t) d t
$$

Use the Jitterbug to calculate the value of object function under fixed time delay, drew the $\mathrm{J}^{\prime} \mathrm{s}$ relationship between the sample period and time delay, the result is shown in Fig.4.

The control system uses PD controller $(\mathrm{K}=1.5, \mathrm{Td}=0.035)$, and in order to make NCS' s time delay and shake more distinct, the gap of sample time is $\mathrm{h}=10 \mathrm{~ms}$. In the graph the $\mathrm{x}$ and $\mathrm{y}$ axes represent NCS network delay and sample period respectively, $z$ axis represents the performance function of 
system is J. From the graph we can figure out that as the time delay increases $\mathrm{J}$ is getting larger. The sample period is $8 \mathrm{~ms}$, without a time delay, $\mathrm{J}<2$; but when the time delay takes up $60 \%$ of the time period, $\mathrm{J}$ will become infinite and then causes the instability of the system. It is obvious that the sample period and the variation of the time delay influence the system' s characteristic a lot.

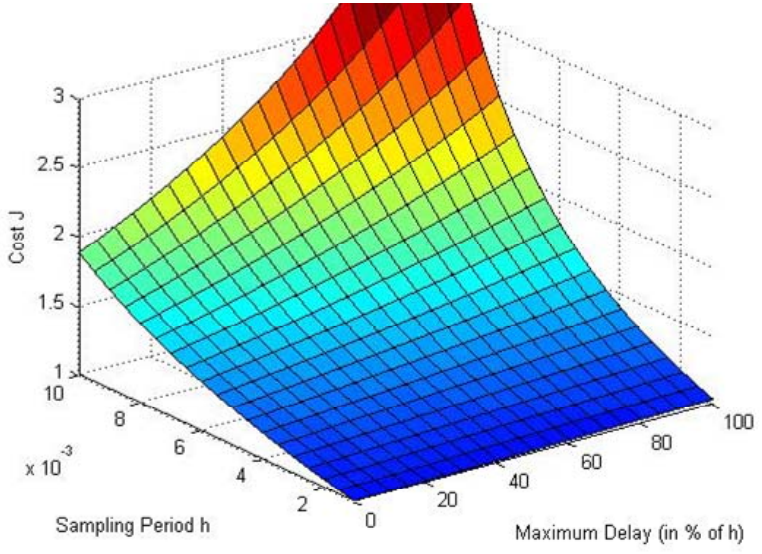

Fig. $4 \mathrm{~J}$ in random time delay

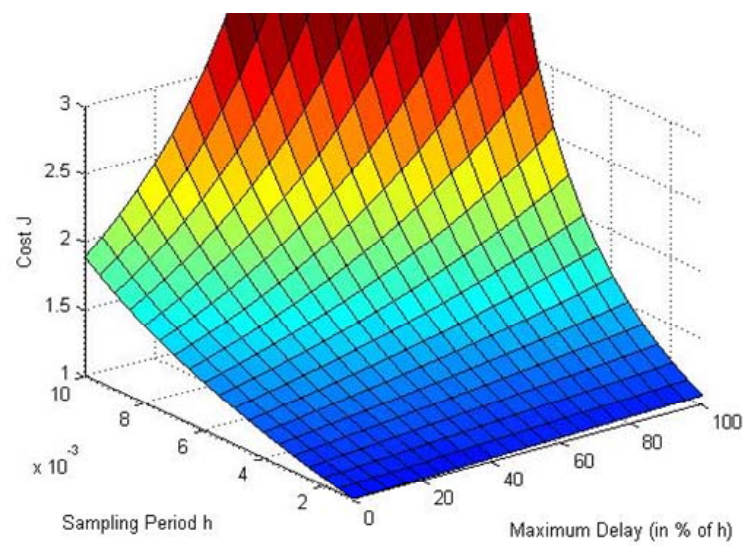

Fig.5 $\mathrm{J}$ in fixed time delay

In the internet based control system, the CSMA/CD protocol makes the time delay random, at the receiving end, the buffer is used to deal with the random delay, but it will lead to the selection of the parameters of the system tend to be conservative. Use the Jitterbug to calculate NCS performance index function value $\mathrm{J}$ in fixed time delay, the outcome is drawn as Fig.5. As it is studied, with the enlargement of the unstable system area, it is mainly caused by the worst condition of the Internet time delay database.

Use Jitterbug can analysis the compensate strategy of the network time delay. Adjust the controller parameter to make adjustment with the output. The reference experience or the time delay data and control parameter are stored in the chart. In the working process the controller decides the $\tau_{s c}$ first and estimate the total time delay of the control system by $\tau_{s c}$, and then utilize $\tau_{s c}$ to look for the parameters in the chart, output the calculated signals through the controller to realize the control of the system. To make compensate of the systems above, offset parameters are used as shown in Fig.1. The system performance index curve after time delay compensation is shown in Fig.6.Compared with Fig.4, the performance index function of the system can be limited by the system compensation. The system performance is improved.

Table 1. Parameter adjustment table

\begin{tabular}{ccccccc}
\hline & 1 & 2 & 3 & 4 & 5 & 6 \\
\hline $\begin{array}{c}\text { Time delay } \\
\text { (ms) }\end{array}$ & 0 & 0.035 & 0.045 & 0.055 & 0.065 & 0.075 \\
$\begin{array}{c}\text { Ratio } \\
\text { Coefficient }\end{array}$ & 1.5 & 1.2 & 1.1 & 0.98 & 0.86 & 0.78 \\
$\begin{array}{c}\text { Differential } \\
\text { quotient }\end{array}$ & 0.035 & 0.04 & 0.042 & 0.046 & 0.049 & 0.052 \\
\hline
\end{tabular}

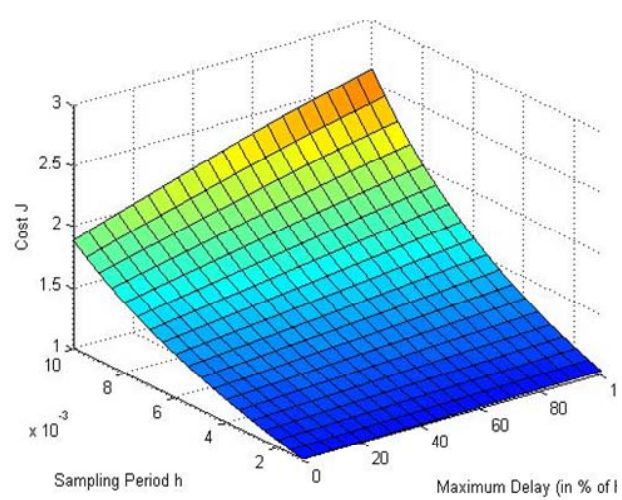

Fig.6 J after time delay compensation

\section{Distributed Real Time Performance Simulation Research}

\subsection{Simulation model of network control system based on TrueTime}

The TrueTime tool box is a network control simulation one invented by Dan Henriksson and Anton Cervin from Sweden, which can do a comprehensive simulation study on the network delay, network parameters of the system performance, the control method, network scheduling and other aspects. Construct a NCS simulation system based on TrueTime, take the DC motor as the controlled object. 
The system utilizes four modules include the sensor module, actuator module, disturbance module, and communication process to build the close-loop simulation model of network control system.

The network DC motor servo process can be described by a continuous time transfer function:

$$
G(s)=\frac{1000}{s(s+1)}
$$

Discrete PID controller as following:

$$
\begin{aligned}
& P(k)=K(r(k)-y(k)) ; I(k+1)=I(k)+\frac{K h}{T_{i}}(r(k)-y(k)) ; D(k)=a_{d} D(k-1)+b_{d}(y(k)-y(k-1)) \\
& u(k)=P(k)+I(k)+D(k) ; a_{d}=\frac{T_{d}}{N h+T_{d}} ; b_{d}=\frac{N K T_{d}}{N h+T_{d}}
\end{aligned}
$$

In which, $K$ represents the ratio coefficient, $T_{i}$ represents the integral coefficient, $T_{d}$ represents the differential coefficient, $h$ represents the sampling period.

\subsection{Control performance influence by interference of network communication}

Initialized the sensor as time driven, the actuator and controller were set as event driven, the controller was set to use PID control, the time driven sensor collected the data of the controlled object and transmits the data to the network and returned the execution of the controlled object through the network. The interference node in the simulation model, which belongs to the high priority task, is used to interfere the network communication.

The controller used PD control, and the initial setting parameters were Sampling period $\mathrm{h}=0.010 \mathrm{~s}$, ratio coefficient $\mathrm{K}=1.5$, integral coefficient $\mathrm{Td}=0.035$, used the $\mathrm{RM}$ network scheduling. Control performance influence by interference of network communication with and without interference were shown in Fig. 8. The peak in the dispatch diagram indicated that the thread is running, the intermediate value represents the task being seized, The lowest value indicates that the task is in a state of sleep. From the Figs below, the scheduling of the system can be judged and it provides the system performance evaluation with a reliable basis.
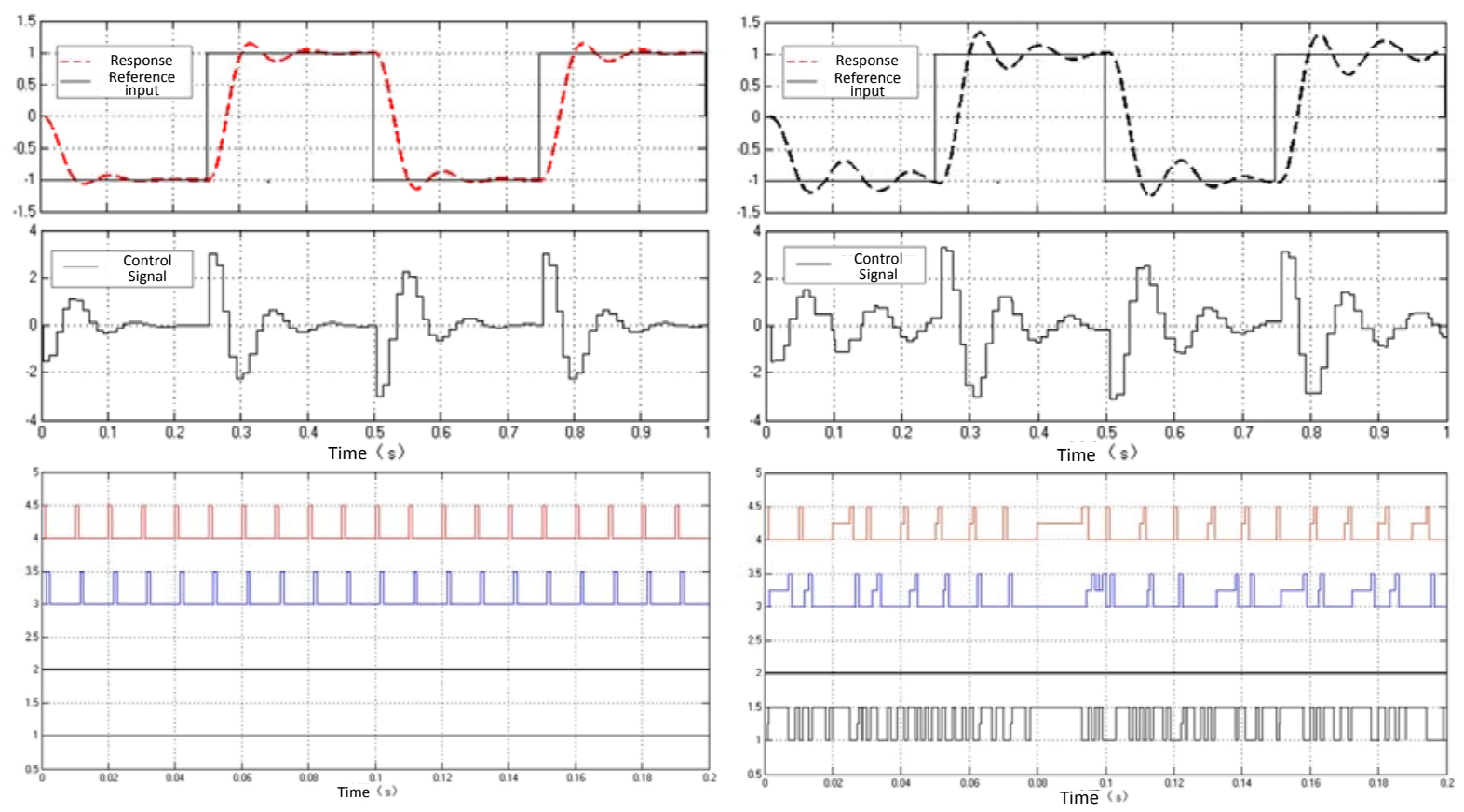

(a)Control under no Interference

(b) Control under Interference communication

Fig. 8 Control performance influence by interference of network communication

As what have been studied, in the absence of interference, the output of the Y is transmitted through the network and quickly tracks the change curve of the reference input R.PD control method can be used for fast and effective regulations, and the control performance of the system is stable. When the interference signal is added to the controller and communication process, though the 
control performance of the system is obviously decreased, the control strategy can still be adjusted effectively.At this time, the network scheduling simulation module will adjust the interfering nodes. So the network scheduling strategy determines the execution differences between the network and the computer and influences the stability of the system. So it must be designed reasonably by the features of tasks.

\subsection{The impact of network Scheduling Strategies for the control performance}

NCS models of three DC motors are established using TrueTime for research the network scheduling methods. The sampling periods of the three motors were respectively0.006s, $0.005 \mathrm{~s}$, $0.004 \mathrm{~s}$, kept the other simulation models unchanged, used RM and EDF scheduling strategies respectively, the simulation results and scheduling curves are shown in Fig.9 and Fig.10.
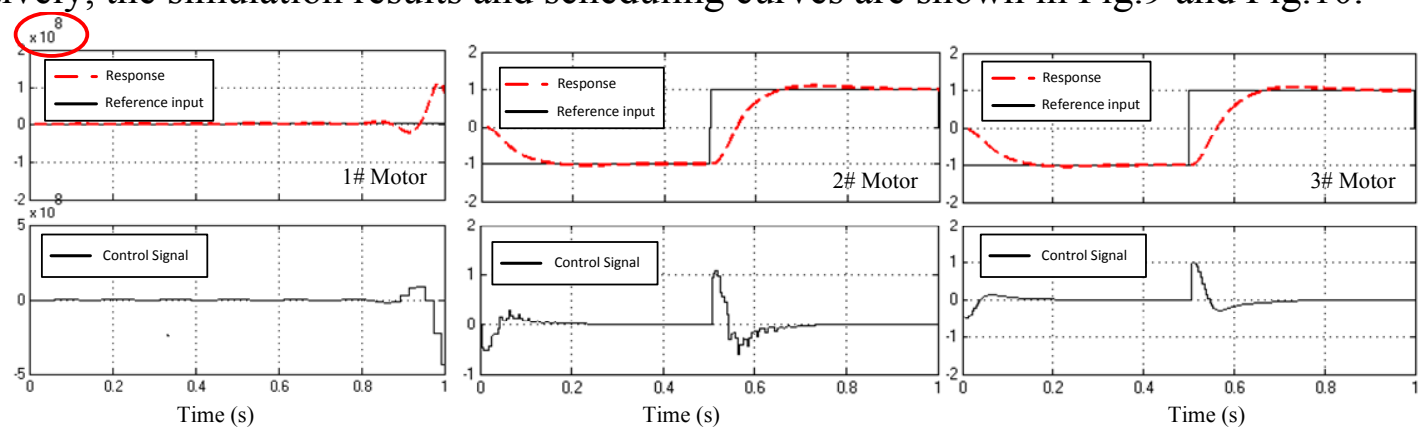

(a) Using the flat rate RM scheduling strategy
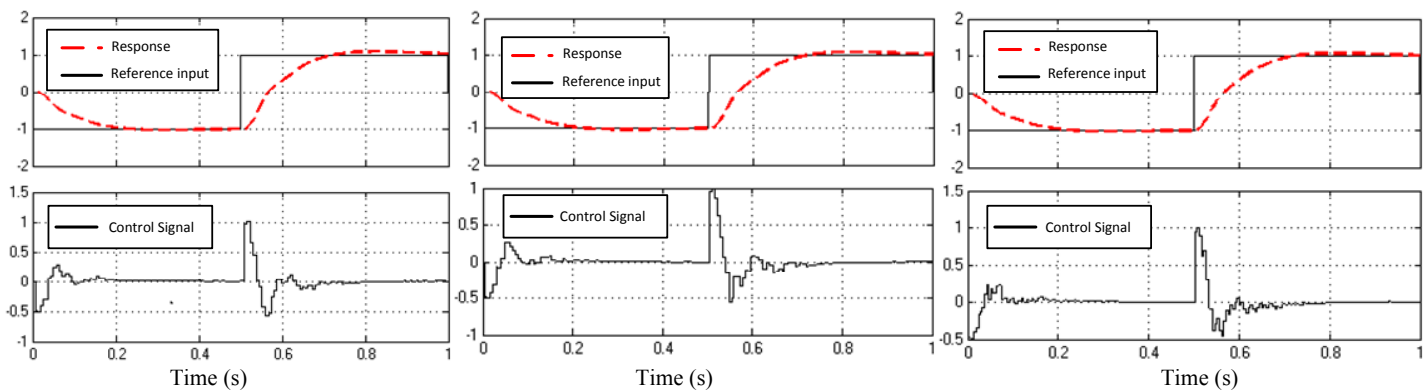

(b) Using the smallest deadline EDF scheduling priority strategy

Fig.9 Comparison of the motor operating performance using the RM and EDF Scheduling Strategies

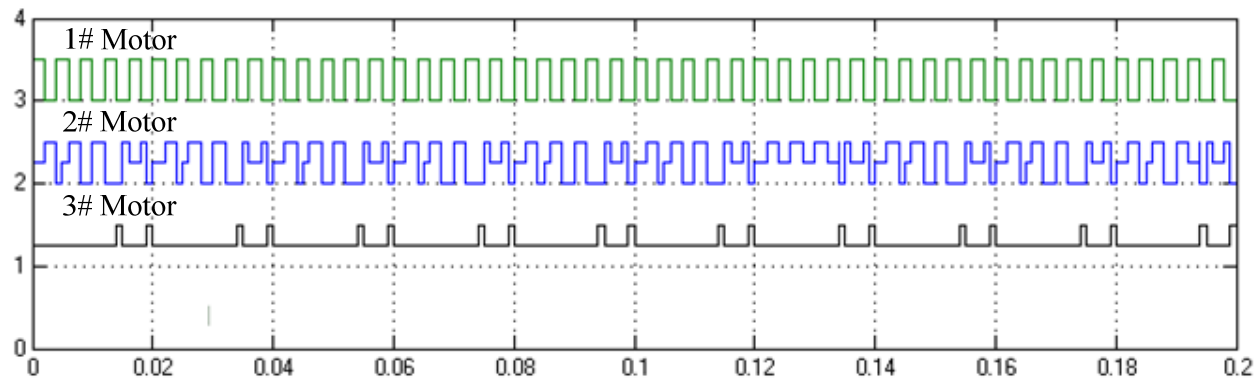

(a) Control signal curve using RM scheduling strategy

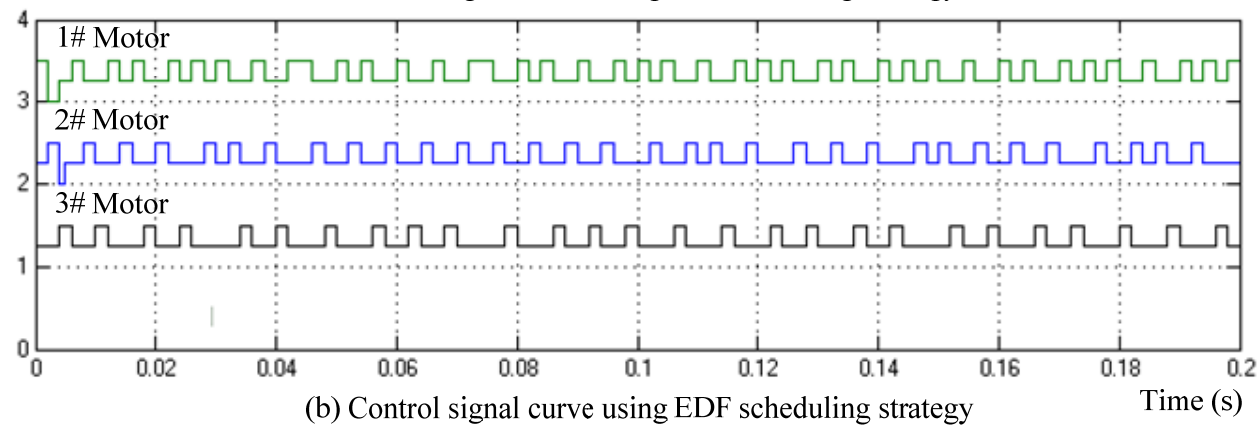

Fig.10 Comparison between RM and EDF scheduling curves

In Fig. 9 and Fig.10, when a RM scheduling strategy was used, the low priority of motor1 is firstly being occupied, and there' s no feedback signal, which caused the instability of the system. When the 
EDF was used, dynamic allocations of priority according to the length of time limit for task distance was being used, so each task was able to be scheduled in a reasonable way. Control performances of the three motors were stable.

\section{Summary}

This paper did researches on the time delay of the network control system, the influence of sampling rate and information transfer interval on the stability of the system. Combined with the experiments of coal mine equipment, utilized simulation method to study the performance of system, and has set a reference for choosing the best parameters of the coal mine distributed control system. And came to the following conclusions:

(1) The network time delay can be rectified by constructing a reasonable observer, but it is based on using the time driver of the sensor which causes unnecessary network resource usage due to the sampling rate and information transmission time interval. This will badly hurt the stability of the system. So it must be optimized before being used.

(2)In the network control system development, static analysis and dynamic testing methods were adopted in the analysis of the comprehensive evaluation strategy, which is very effective to determine the optimal operating parameters of the system.

(3) The network simulation takes the network communication in to account and provided it with a more convenient and effective analysis method. The simulation bench does researches in the control object, actuator, sensor and controller, which is helpful for determining the optimal operating parameters of the system and insuring the real-time function of the network communications.

\section{Acknowledgements}

This work was financially supported by the National Natural Science Foundation Project (U1361121), China Postdoctoral Science Foundation (2015M582692), Scientific Research Program Funded by Shaanxi Provincial Education Department (14JK1473), Shannxi Postdoctoral Science Foundation (No.201406) and Xi'an Collaborative Innovation Program (CXY1519).

\section{Reference:}

[1] Meng Huixia. Research on Network Communication of Remote Monitoring and Control System in Coal Mine. Coal Engineering, No.7 (2010), p.99-101.

[2] Du Dajun, Fei keen, Song Yang, Li Xue. A brief review and Prospect of network control system. Journal of Instrumentation, No.03 (2011), p.713-721.

[3] Chen Zaiping, Yue Youjun. Industrial control network and field bus technology. Beijing: mechanical industry press, 2006.

[4] Wu Xiaoping. Research on network control system based on time delay compensation and generalized predictive control algorithm. Wuhan University of Science and Technology, 2007.

[5] Xiao Yong, Zhang Jun, Yan Jiaguang. Design and implementation of CAN bus network control system in coal mine monitoring system. mining safety and environmental protection, No.06 (2008), p.34-35+42.

[6] Fu Lei, Shi Wei, Mei Bu, Jinye. PROFINET Industrial Ethernet Design Automation System Based on. continuous casting of heavy machinery, No.02(2012), p41-44.

[7] Yue Dong, Peng Chen, Han. Qinglong. Network control system analysis and synthesis. Beijing: Science press, 2007

[8] Walsh G.C., Hong Ye, ushnell L.G. Stability analysis of networked control systems. IEEE Transactions on Control Systems Technology. Vol.10, No3(2002), p438-446. 